\title{
Tuberculoma hepático que simula metástasis de carcinoma neuroendocrino gástrico
}

\author{
Hepatic tuberculoma simulating metastasis of gastric \\ neuroendocrine carcinoma
}

\author{
María Fernanda Anduaga-Peña ${ }^{1,2}$, José E. Quiñones-Sampedro², Manuel J. Iglesias-Iglesias², \\ María José Servide-Staffolani ${ }^{1}, 3$, Luis Miguel González-Fernández², \\ María del Carmen Esteban-Velasco ${ }^{2}$, Luis Muñoz-Bellvis ${ }^{3}$, Patrizio Petrone ${ }^{1,4}$ \\ 1 Department of Surgery, NYU Winthrop Hospital, Mineola, Nueva York, Estados Unidos \\ 2 Hospital Universitario de Salamanca. Salamanca, España. \\ 3 Hospital Universitario de Cruces, en Barakaldo. Vizcaya, España. \\ 4 Clinical Associate Professor of Surgery, New York Medical College, New York, USA \\ Presentado en la xx Reunión Nacional de Cirugía de la Asociación Española de Cirujanos (AEC). 21 a 23 de octubre de 2015, \\ Granada, España.
}

\section{Resumen}

La tuberculosis es una de las enfermedades infecciosas más frecuentes de distribución mundial, con una amplia presentación clínica. La afectación hepática de la tuberculosis es poco frecuente, pero más infrecuente aún lo es el tuberculoma hepático. Se presenta el caso de una paciente con tuberculoma hepático solitario que simula metástasis hepática secundaria a tumor neuroendocrino.

Palabras clave: mycobacterium tuberculosis; tuberculosis; tuberculosis hepática; tuberculoma; hiperplasia nodular focal.

\begin{abstract}
Tuberculosis is one of the most common infectious diseases, with worldwide distribution and ample clinical manifestations. Hepatic tuberculosis is rare but hepatic tuberculoma is even rarer. We report an unusual case of a solitary hepatic tuberculoma simulating hepatic metastasis of a neuroendocrine tumor.
\end{abstract}

Key words: mycobacterium tuberculosis; tuberculosis, hepatic; tuberculoma; focal nodular hyperplasia.

\section{Introducción}

La tuberculosis es una entidad infecciosa común y bien descrita en la literatura, distribuida predominantemente en países en vía de desarrollo ${ }^{\perp}$. La afectación hepática en la tuberculosis extrapulmonar es poco frecuente y puede debutar de diferentes formas clínicas, entre ellas la más frecuente es la secundaria a tuberculosis miliar ${ }^{1-3}$. Dentro de las manifestaciones poco frecuentes se encuentra el tuberculoma hepático solitario?3. Dado que esta presentación es poco común y los síntomas son inespecíficos, el diagnóstico se dificulta sin un análisis patológico que lo confirme. No es infrecuente, como en el caso que se expone, que

Fecha de recibido: 26/06/2017 - Fecha aceptación: 21/11/2017

Correspondencia: Patrizio Petrone, Director of Surgical Research Program Director, International Visiting Scholars and Research Fellowship Department of Surgery - NYU Winthrop Hospital Clinical Associate Professor of Surgery, New York Medical College 222 Station Plaza North, Suite 603, Mineola, Long Island, New York 11501 - USA. Correo electrónico: patrizio.petrone@gmail.com, patrizio.petrone@nyulangone.org

Citar como: Anduaga-Peña MF, Quiñones-Sampedro JE, Iglesias-Iglesias MJ, Servide-Staffolani MJ, González-Fernández LM, EstebanVelasco MC, Muñoz-Bellvis L, Petrone P. Tuberculoma hepático que simula metástasis de carcinoma neuroendocrino gástrico. Rev Colomb Cir. 2018;33:307-10. https://doi.org/10.30944/20117582.76 
esta patología se confunda con lesiones malignas hepáticas primarias o secundarias ${ }^{4}$.

Se presenta el caso clínico de una paciente con tuberculoma hepático solitario que simula metástasis de carcinoma neuroendocrino gástrico.

\section{Caso clínico}

Paciente de sexo femenino, de 75 años de edad, con antecedentes personales de hipertensión arterial, colecistectomía y gastrectomía total con esofagectomía distal por tumor neuroendocrino. En seguimiento por el servicio de Oncología, clínicamente asintomática y con examen físico normal. Se realizó una tomografía computarizada (TC) abdomino-pélvica de control a los dos años, en la que se objetivó una lesión de ocupación hepática en segmento IVb, hipervascularizada, de $7 \mathrm{~mm}$, sugerente de metástasis (figura I). Se complementó el estudio con una resonancia magnética (RM) de hígado, la cual mostró aumento de señal en T2 que insinuaba el mismo diagnóstico. Los estudios analíticos complementarios estaban dentro de los parámetros normales. Se decidió realizar

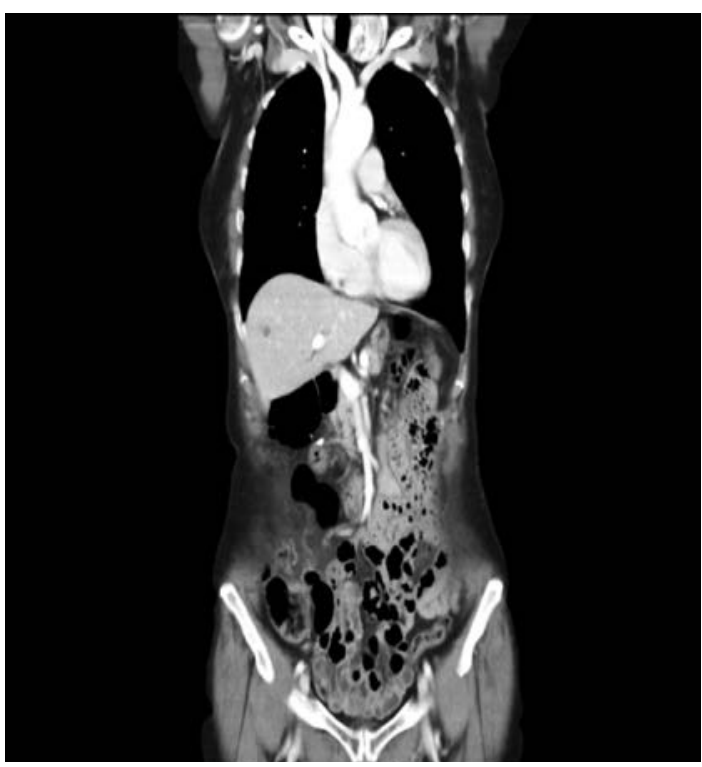

A. Corte coronal. una intervención quirúrgica programada con intención curativa.

Se practicó una resección no anatómica del segmento IVb. Tuvo evolución postoperatoria favorable, y se le dio el alta a las 72 horas. El análisis anatomo-patológico de la pieza fue informado como "granuloma necrotizante con abundantes bacilos ácido-alcohol resistentes compatibles con micobacterias" (figura 2).

El seguimiento estuvo a cargo del Servicio de Cirugía General en conjunto con el Servicio de Enfermedades Infecciosas, pautándose tratamiento con terapia antituberculosa (isoniacida, rifampicina, pirazinamida y etambutol) por el término de nueve meses. Durante el tiempo de seguimiento la paciente no fue bacilífera ni presentó síntoma alguno de enfermedad, y debido a ello no se pudo demostrar otra fuente infecciosa.

\section{Discusión}

La tuberculosis hepática es considerada como una manifestación rara de la tuberculosis abdominal que constituye el $\mathrm{I} \%$ de todos los casos de tuberculosis, usualmente asociada a infección

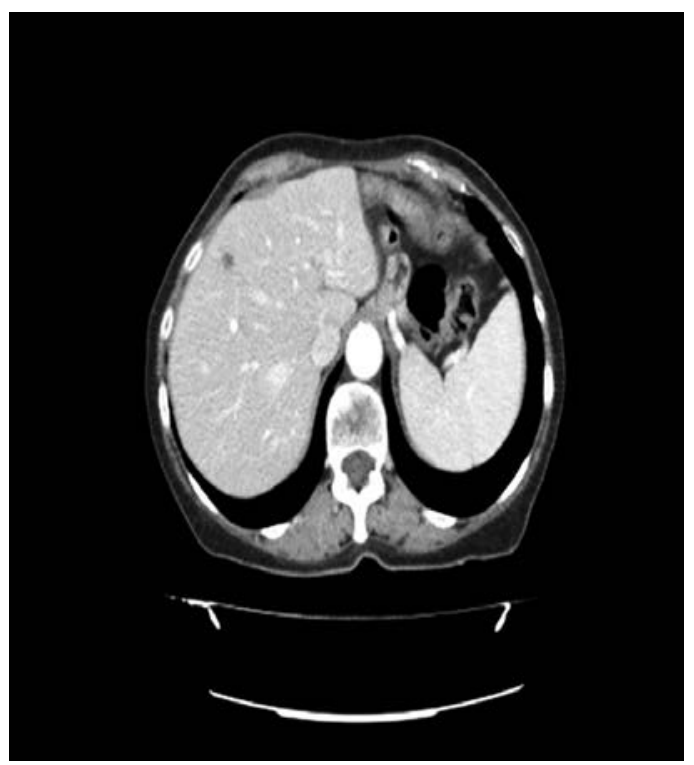

B. Corte axial.

Figuras 1 A y B. Tomografía computarizada abdomino-pélvica en la que se observa una lesión de ocupación hepática en segmento IVb hipervascularizada. 


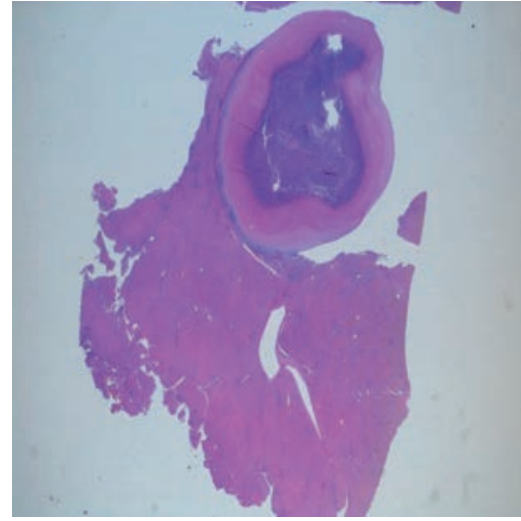

A: Coloración hematoxilina eosina; $x 40$.

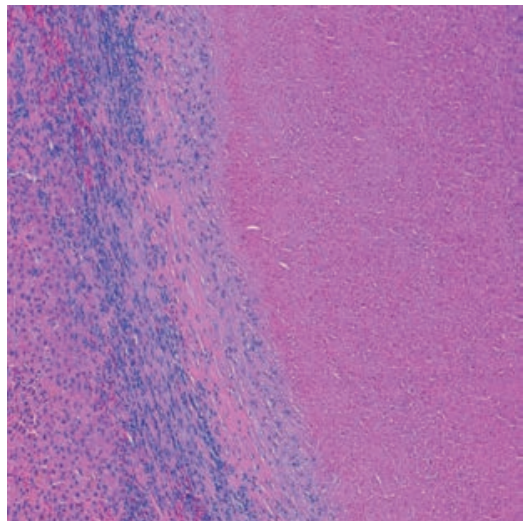

B: Coloración Hematoxilina eosina; x10.

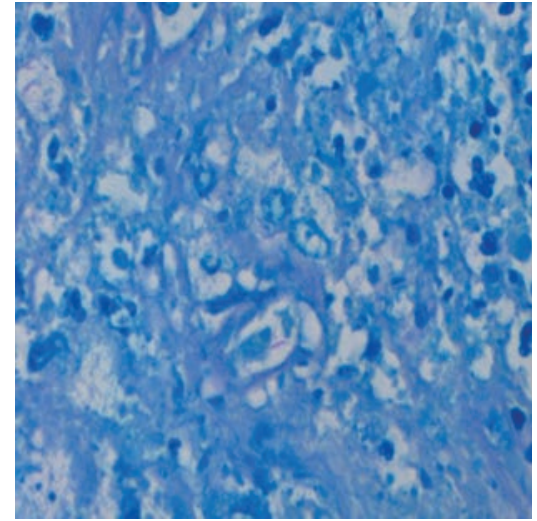

C: Coloración Ziehl-Neelsen; x100.

Figura 2A, B y C. Granuloma necrosante con abundantes bacilos ácido-alcohol resistentes compatibles con micobacterias.

pulmonar o del aparato digestivo. Existen tres formas de presentación: afectación hepática difusa asociada a enfermedad pulmonar (80\% de los casos), afectación hepática difusa sin compromiso pulmonar, y finalmente afectación local como absceso o tuberculoma solitario. Esta última forma de presentación es muy poco frecuente, incluso en áreas endémicas ${ }^{\mathrm{I}-3}$.

El primero en describir el compromiso macronodular de la tuberculosis en el hígado fue Bristowe $^{4}$ en I858, al encontrar lesiones cavitadas en I2 de I67 necropsias con lesiones tuberculosas en el intestino.

En un estudio realizado en Sudáfrica entre I955 y I98I, con un total de 296 casos de tuberculosis hepática, solo I,3\% presentó esta manifestación clínica5. Suele presentarse con síntomas muy inespecíficos como fiebre o febrícula, pérdida de peso, dolor abdominal en epigastrio y en hipocondrio derecho, hepatomegalia (esporádicamente), elevación de la fosfatasa alcalina (determinada en pruebas de laboratorio), o simplemente puede presentarse como hallazgo incidental en un estudio anátomo-patológico del hígado $o^{6,7}$, como sucedió en este caso.

El diagnóstico de tuberculoma hepático no es sencillo. Suele ser intra- o postquirúrgico mediante análisis de la muestra tomada a través de laparotomía o laparoscopia en el estudio de una masa hepática. El hallazgo del bacilo de Koch en el material obtenido por biopsia con aguja fina o quirúrgica, es específico pero raramente reportado.

Los tuberculomas son nódulos con diámetro superior a los $2 \mathrm{~mm}$, aunque puede alcanzar varios centímetros ${ }^{8}$. Las pruebas de imagen para el estudio de tuberculosis hepática son inespecíficas y usualmente se sospechan como lesiones primarias o secundarias ${ }^{4,7}$, como en el caso que se expone. Un estudio complementario que tal vez pudiera haber contribuido en el diagnóstico de este caso es la tomografía por emisión de positrones (PET-TAC) debido al antecedente de tumor neuroendocrino. No se evidenció en ningún momento tuberculosis extrahepática debido a que las pruebas de imagen sugerían que se trataba de una patología secundaria a un tumor neuroendocrino primario. Una vez confirmado el diagnóstico el pilar más importante del tratamiento de esta patología es la terapia antituberculosa, ocasionalmente complementada con tratamiento quirúrgico9. Con la terapia específica el pronóstico es favorable y las lesiones se resuelven en la mayoría de los casos entre 6 y I 8 meses $^{8}$.

\section{Conclusión}

El tuberculoma hepático solitario es la forma más infrecuente de tuberculosis abdominal. En ausencia de síntomas sistémicos es difícil sospecharla y más aún diagnosticarla, dado que las pruebas de imágenes convencionales carecen de especificidad para hacer un diagnóstico diferen- 
cial. Lo habitual es que dicha patología simule una tumoración hepática primaria o secundaria, se establezca el tratamiento quirúrgico y se llegue al diagnóstico de manera diferida. En estos casos el manejo conjunto con el Servicio de Enfermedades Infecciosas es fundamental para completar la terapia antituberculosa.

\section{Referencias}

I. Ardiles V, Rossi G, Acuña J, Romero R, Mullen E, López M, Santibañes E. Tuberculoma hepático: presentación de casos y revisión bibliográfica. Acta Gastroentereol Latinoam. 2004;34:2I-6.

2. Tapias-Vargas LF, Santamaria CM, Tapias-Vargas L, Tapias L. Perforación de íleon terminal y ciego causada por tuberculosis intestinal en un paciente positivo para VIH. Rev Colomb Cir. 20I0;25:332-40.

3. Kharrasse G, Soufi M, Berekhli H, Intissar H, Bouziane M, Ismailli Z. Primary tuberculoma of the liver:
A case report and literature review. Pan African Med J. 20I4;I9:32I. doi: IO.II604/pamj.20I4.19.32I.5IO7.

4. Bristowe T. On the connection between abscess of the liver and gastrointestinal ulceration. Trans Path Soc. I958;9:24I.

5. Zipper R, Rau J, Ricketts R, Bevans L. Tuberculous pseudotumors of the liver. Am J Med. 1976;6I:946-5I.

6. Kawamori Y, Matsui O, Kitaagawa K, Kadoya M, Takashima T, Yamahana T. Macronodular tuberculoma of the liver:: CT and MR findings. Am J Roentgenol. I992;I58:3II-3.

7. Culafic D, Boric I, Vojinovic V, Zdrnja M. Hepatic Tuberculomas. A case report. Rom J Gastroenterol. 2005;I4:7I-4.

8. Cecchini E, Nishimura M, Andreolli M. Tubeculosis hepática. Quirón, 2000;31:I0-5.

9. Hsieh TC, Wu YC, Hsu CN, Yang CF, Chiang IP, Hsieh $\mathrm{CY}$, et al. Hepatic macronodular tuberculoma mimics liver metastasis in a patient with locoregional advanced tongue cancer. J Clin Oncol. 20II;29:64I-3. 